\title{
PRIMER REGISTRO DE ABLABESMYIA (KARELIA) CINCTIPES (DIPTERA, CHIRONOMIDAE) PARA CUBA
}

\author{
Orestes C. Bello González \\ Centro de Investigaciones de Medio Ambiente de Camagüey. Cisneros 105 altos e/Pobre y Angel. \\ Camagüey1 CP: 70 100,Cuba. orestes@cimac.cu
}

\section{RESUMEN}

Se cita por primera vez para Cuba a Ablabesmyia (Karelia) cinctipes (Johannsen, 1946). Las larvas fueron encontradas asociadas a la vegetación acuática en una laguna somera y criadas en condiciones de laboratorio.

Palabras clave: Ablabesmyia (Karelia) cinctipes, Diptera, Chironomidae, primer registro, Cuba.

Title: First record of Ablabesmyia (Karelia) cinctipes (Diptera, Chironomidae) from Cuba.

\section{ABSTRACT}

Ablabesmyia (Karelia) cinctipes (Johannsen, 1946) is recorded be first time from Cuba. The larvae were found associated to aquatic vegetation in a shallow lagoon and reared under laboratory conditions.

Key words: Ablabesmyia (Karelia) cinctipes, Diptera, Chironomidae, first record, Cuba.

El género Ablabesmyia Johannsen, 1905 (Diptera, Chironomidae) ha sido mencionado para Cuba en inventarios faunísticos de algunos embalses distribuidos por todo el país, incluyendo la Isla de la Juventud (Poddybnaya et al., 1976; Biochino, 1976; Plasencia, 1987; Plasencia y Calzadilla, 1987) aunque no aparece referido en los compendios de Alayo y García (1983) y Alayo y Garcés (1989). Los estudios en los que se menciona no refieren especie alguna, probablemente debido a que la identificación de los ejemplares de Ablabesmyia es muy difícil sin tener en cuenta las fases de larva y pupa relacionadas (Oliveira y Fonseca-Gessner, 2006). Ablabesmyia (Karelia) cinctipes (Johannsen, 1946), descrita a partir de ejemplares de La Florida (EUA) se distribuye además, en otras localidades de EUA (Hudson et al., 1990), América Central, algunas de las Antillas Menores (Spies y Reiss, 1996) y Bahamas (Roback, 1970). Los estadíos inmaduros de la especie fueron descritos por Caldwell (1993); este autor también ubicó los caracteres de la larva y la pupa en la clave de Roback (1985) para los estadíos inmaduros de Ablabesmyia. Este trabajo representa el primer registro de $A$. cinctipes para Cuba.

Las larvas fueron colectadas en una laguna temporal sobre sustrato geológico ultramáfico, en el momento de la colecta la laguna tenía $0.6 \mathrm{~m}$ de profundidad. Las larvas se encontraron asociadas a Ludgiwia leptocarpa (Nutt.) H. Hara, 1953. Fueron transportadas en frascos con agua del hábitat y criadas en el laboratorio ubicándolas independientemente en pocetas de cría de cristal de aproximadamente $5 \mathrm{~cm}^{3}$ en las que se depositó un fragmento de hoja muerta. Las larvas fueron alimentadas con larvas de Chironomus sp de $2^{\text {do }}$ y $3^{\text {er }}$ instar capturadas en la misma laguna. Todas las larvas puparon y los adultos emergieron entre los 6 y los 8 días siguientes a su captura. La piel de cada larva con su correspondiente exubia pupal fue montada en un mismo portaobjetos utilizando Euparal. Las preparaciones fueron observadas utilizando un microscopio de campo brillante y aumentos de hasta $1000 \mathrm{X}$. 
Para la identificación de la especie nos basamos en el trabajo de Caldwell (1993). La combinación de los siguientes caracteres, todos presentes en los ejemplares estudiados, permite una certera identificación de $A$. cinctipes: en el estadío larval, la presencia de tres garras oscuras (dos cortas y anchas y una más alargada y estrecha) en los parápodos posteriores (Fig. $1 \mathrm{~A}$ ), longitud del segmento antenal I entre 457 y $496 \mu \mathrm{m}$, del segmento antenal II entre 89 y $94 \mu \mathrm{m}$, del segmento I del palpo maxilar (P1) entre 40 y $47 \mu \mathrm{m}$, del segmento II del palpo maxilar (P2) entre 44 y $54 \mu \mathrm{m}$, cociente P1/P2 entre 0.86 y 0.95 y longitud de la mandíbula entre 164 y $173 \mu \mathrm{m}$. En el estadío pupal el patrón de coloración del estuche alar (Fig. 1 B) y el color uniforme del terguito IV (Fig. 1 C).

Material examinado: 3 pieles de larvas, 3 exubias pupales, relacionadas mediante la cría. Meseta de San Felipe: $21^{\circ} 38^{\prime} 37^{\prime \prime}$ N, $78^{\circ} 00^{\prime} 15^{\prime \prime}$ W, Esmeralda, Camagüey, noviembre, 2010. O. Bello. Los especímenes se encuentran depositados en la colección del Centro de Investigaciones de Medio Ambiente de Camagüey, una dependencia del Ministerio de Ciencia, Tecnología y Medio Ambiente.
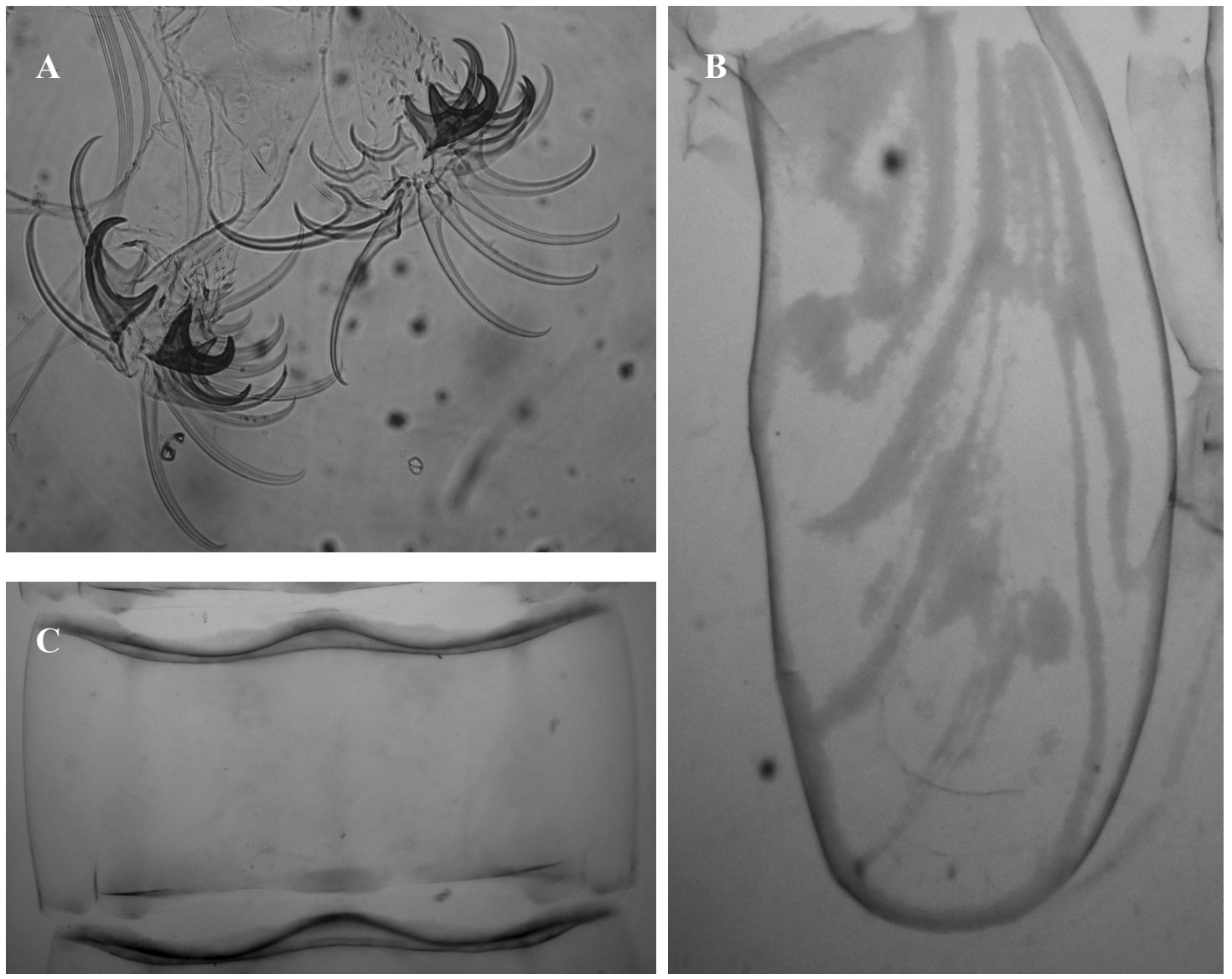

Figura 1. Partes de Ablabesmyia (Karelia) cinctipes. A, garras de los parápodos posteriores de la larva. B, estuche alar de la pupa. C, terguito IV de la pupa. 


\section{AGRADECIMIENTOS}

A Eddy Martínez Quesada MSc, por la identificación de la planta acuática.

\section{LITERATURA CITADA}

Alayo, P. D. y G. Garcés. 1989. Introducción al estudio del Orden Diptera en Cuba. Editorial Oriente, Santiago de Cuba, 223 pp.

Alayo, P. D. e I. García. 1983. Lista anotada de los dípteros de Cuba. Editorial CientíficoTécnica, Habana, 204 pp.

Biochino, G. I. 1976. Caracterización de la fauna de la vegetación de los embalses de Cuba. Serie Forestal (33): 1-6.

Caldwell, B. A. 1993. The inmature stages of Ablabesmyia cinctipes (Johannsen) with comments on ecology. Spixiana, 16 (1): 49-52.

Hudson, P. L., D. R. Lenat., B. A. Caldwell y D. Smith. 1990. Chironomidae of the Southeastern United States: A checklist of species and notes on biology, distribution and habitat. United States Fish and Wildlife Service. Fish and Wildlife Research, (7): 1-46.

Oliveira, C. S. N. y A. A. Fonseca-Gessner. 2006. New species of Ablabesmyia Johannsen (Diptera, Chironomidae, Tanypodinae) from the Neotropical Region, with description of male adults and immature stages. Rev. Bras. Zool., 23 (3): 740-745.

Plasencia, J. M. F. 1987. La fauna béntica y perifítica en el embalse La Fé. Reporte de investigación del Instituto de Ecología y Sistemática, (47): 1-9.

Plasencia, J. M. F. y R. L. Calzadilla. 1987. La fauna del bentos en tres embalses de la Isla de la Juventud. Reporte de investigación del Instituto de Ecología y Sistemática, (50): 1-24.

Poddybnaya, T .L., T. N. Kurashkovskaya, V. I. Taranova y V. I. Mitropolski. 1976. Datos sobre la fauna del fondo de los embalses de Cuba. Serie Forestal, (32): 1-6.

Roback, S. S. 1970. Adults of the Subfamily Tanypodinae (Pelopinae) in North America (Diptera Chironomidae). Monographs of The Academy of Natural Sciences of Philadelphia, 17: $1-410$.

Roback, S. S. 1985. The immature Chironomids of the eastern United States.VI. Pentaneurinigenus Ablabesmyia. Proceedings of the Academy of Natural Sciences of Philadelphia, Philadelphia, 137 (2): 153-212.

Spies, M. y F. Reiss. 1996. Catalog and bibliography of Neotropical and Mexican Chironomidae (Insecta, Diptera). Spixiana Suppl., 22: 61-119.

[Recibido: 5 de Julio, 2011. Aceptado para publicación: 10 de enero, 2012] 